\title{
Safety of combined lumbar and sacral plexus block in high risk patients undergoing fractured neck of femur repair under general anaesthesia
}

\author{
T. Dixit, A. Banerjee, S. Singh \\ Royal Liverpool University Hospitals, Liverpool, United Kingdom.
}

Correspondence: Shiv Kumar Singh. Address: Anaesthesia Office 11th Floor, Royal Liverpool University Hospitals, Prescot Street Liverpool L7 8XP, the United Kingdom. Email: shvkmrsngh@aol.com

Received: February 10, 2014

Accepted: July 6, 2014

Online Published: July 29, 2014

DOI : $10.5430 /$ crim.v1n2p221

URL: http://dx.doi.org/10.5430/crim.v1n2p221

\begin{abstract}
In this case series we present three high-risk patients with multiple co-morbidities listed for repair of fractured neck of femur with hemiarthroplasty under general anaesthesia. All three were administered combined unilateral lumbar plexus block (LPB) and sacral plexus block (SPB) for intra and post-operative analgesia. One patient presented with severe chronic obstructive pulmonary disease in combination with severe aortic stenosis. The second one had extensive medical history, was in fast atrial fibrillation (AF) and was actively taking clopidogrel. The last patient had an automatic implantable cardioverter defibrillator (AICD) for dilated cardiomyopathy secondary to ischaemic heart disease and was in left ventricular failure. All three patients had uneventful recovery and were discharged to the ward following their initial stay in the critical care unit. We discuss the evidence and controversies surrounding the techniques employed in these three difficult cases.
\end{abstract}

\section{Keywords}

Lumbar plexus block (LPB), Sacral plexus block (SPB), Local anaesthesia, Nerve block, Anticoagulants, Antiplatelets, Clopidogrel, Pain relief

\section{Case presentations}

\subsection{Case 1}

An 80 year old female, weighing 75kg was admitted with a right sided fractured neck of femur following a fall at home. She was known to have severe chronic obstructive pulmonary disease (COPD) requiring long-term oxygen therapy at home. She had poor exercise tolerance and was able to walk only 15 yards with the aid of a delta frame. Spirometry revealed FVC of $0.93 \mathrm{~L}$ (67\% predicted), FEV1 0.66L (56\% predicted) and PEFR of 3.21L (39\% predicted). Echocardiogram done to assess her cardiac function revealed critical aortic stenosis and a heavily calcified aortic valve, with a peak gradient of $73 \mathrm{mmHg}$ and aortic valve area of approximately $0.7 \mathrm{~cm}^{2}$. Other additional findings included mild aortic regurgitation, mild concentric left ventricular hypertrophy and moderate mitral regurgitation. Though left ventricle 
systolic function appeared preserved, mild diastolic dysfunction was noted. There was evidence of tricuspid regurgitation with right ventricle systolic pressure of $34 \mathrm{mmHg}$. Peak TR doppler suggested moderate pulmonary artery hypertension.

The patient also had a history of hypothyroidism, recurrent UTIs with the use of a long-term catheter, osteoporosis, hypertension, paroxysmal atrial fibrillation and chronic kidney disease (CKD) stage 3. Her medications included prednisolone, quetiapine, mirtazepine, thyroxine, furosemide, amlodipine, docusate, amiodarone, iron sulphate, calcium supplementation and inhalers. Her surgical history included a left total hip replacement some 6 months prior, under general anaesthesia with a 3:1 block. The previous general anaesthetic and post-op stay was recorded to be relatively uncomplicated.

During this admission the patient was apyrexial with blood pressure of 165/83 and pulse rate $85 \mathrm{bpm}$. Arterial blood gas analysis on admission showed $\mathrm{pH} 7.44, \mathrm{pCO}_{2} 8.33 \mathrm{kPa}, \mathrm{pO}_{2} 6.46 \mathrm{kPa}$, bicarbonate $37.7 \mathrm{mmol}$, $\mathrm{BE}+14.2$ on breathing room air. Admission bloods showed haemoglobin (Hb) 11.1(g\%), WCC $25.0\left(10^{3}\right)$, platelets $295\left(10^{9}\right), \mathrm{Na}^{+} 139 \mathrm{mmol}, \mathrm{K}^{+}$ 4.0mmol, urea 7.5mmol, creatinine 94mmol, normal LFTs, PT 12.8Sec and aPTT 21.8Sec. ECG demonstrated sinus rhythm with evidence of LA enlargement and LVH. On admission she was commenced on oxygen 3L/min via nasal catheter. Intravenous hydrocortisone and amoxicillin were started for a presumed chest infection on the basis of increased sputum production, raised white cell count and left basal shadowing on chest x-ray.

The patient was listed for a right Thompson's hip prosthesis. On anaesthetic pre-op assessment she was graded as American Society of Anaesthetist (ASA) class 4. Her airway assessment was classed as Mallampati grade 1 and on oral examination, she was found to be edentulous. The anaesthetic options were discussed with the patient and the decision was taken to conduct GA with LPB and SPB. Anaesthesia was induced with 100mcg fentanyl and 120mg of propofol and an LMA size 4 was used for the initial airway management. Before positioning her on to the left lateral side for the plexus blocks and the surgery, an arterial line was inserted for continuous blood pressure and ABG monitoring.

The plexus blocks were performed using a 100mm Stimuplex needle using landmark techniques. For the lumbar plexus block, 30ml of $0.375 \%$ Levo-Bupivacaine was infiltrated with a current threshold of $0.3 \mathrm{~mA}$, and for the sacral plexus block, $10 \mathrm{ml}$ of $0.375 \%$ Levo-Bupivacaine was infiltrated again with a threshold of $0.3 \mathrm{~mA}$. There were no immediate complications from the nerve block. Muscle relaxant was administered and trachea was intubated for the procedure due to suspected concurrent chest infection and risk of de-saturation due to mucous plugging. Throughout the procedure, she was ventilated using pressure controlled ventilation (PCV) and anaesthesia was maintained with sevoflurane in air/oxygen mixture. Intra-operatively, she remained haemodynamically stable and a mean arterial pressure (MAP) of 80mmHg was maintained intraoperatively with $1 \mathrm{~L}$ of crystalloid and $1 \mathrm{~L}$ of colloid. Total vasopressors used during the procedure were just 1mg of metaraminol. Other intra-op analgesia included $2 \mathrm{mg}$ of morphine. Following the procedure, which lasted approximately 90 minutes, trachea was successfully extubated. She was transferred to the post operative critical care unit (POCCU) for post-op care. Arterial blood gas (ABG) post-operatively showed $\mathrm{pH} 7.38, \mathrm{pCO}_{2}$ 9.5kPa, $\mathrm{pO}_{2}$ 8.6kPa, bicarbonate $38 \mathrm{mmol}$, base excess +13.2 on $50 \%$ oxygen. She remained comfortable overnight requiring only intravenous paracetamol for analgesia. The following day, two doses of oral codeine (30mg) were administered along with paracetamol for analgesia. No opioids were used for analgesia at any time during her stay in the POCCU. She was discharged from the unit the day following her procedure on $3 \mathrm{~L} / \mathrm{min}$ nasal specs. On the ward, she sat out on day 3 and was successfully mobilised on day 4.

\subsection{Case 2}

A 90-year-old female was admitted following a fall during which she sustained a fractured neck of femur. She had a background of hypertension, chronic kidney disease (CKD) stage-3, previous hip fracture fixation with dynamic hip screw (DHS), bilateral cellulitis and an implanted loop recorder for syncopal episodes. She had poor exercise tolerance of about 20 yards. An echocardiogram in 2004 demonstrated moderate regional impairment of LV function with mild mitral and tricuspid regurgitation. Her medications included ranitidine, irbesartan, simvastatin, amlodipine, clopidogrel and 
alendronate. Clopidogrel was stopped only on admission. In the Emergency Department; her BP was 159/96, pulse rate $110 \mathrm{bpm}, \mathrm{SpO}_{2} 98 \%$ breathing $28 \%$ oxygen and a temperature of $36.2^{\circ} \mathrm{C}$. Her ECG showed atrial fibrillation and her CXR suggested left lower lobe pneumonia for which she was receiving co-amoxiclav. The surgery was initially cancelled on day 1 post admission due to de-saturation in the anaesthetic room and fast AF (rate 160), which failed to resolve with IV amiodarone.

She was admitted to high dependency unit (HDU) for pre-operative optimisation, during which she received a maintenance dose of amiodarone and digoxin for rate control. With better rate control, she was listed for a Thompson's hemiarthroplasty on day 2 post-admission. Pre-operative airway assessment was graded as Mallampati 2 and she was classed as ASA 4. The pre-op bloods showed Hb 11.6 (g\%), platelets $173\left(10^{9}\right), \mathrm{Na}^{+} 126 \mathrm{mmol}, \mathrm{K}^{+} 4.4 \mathrm{mmol}$, Ur $4.2 \mathrm{mmol}$, $\mathrm{Cr}$ 62mmol; clotting screen was within normal limits. Anaesthesia was induced with 100mg propofol, 100mcg fentanyl and an LMA size 3 was inserted for airway management. She was then turned on to her right lateral side for the plexus blocks and the surgery.

A $100 \mathrm{~mm}$ Stimuplex needle was used for the lumbar plexus block and at a threshold current of $0.5 \mathrm{~mA}, 30 \mathrm{ml}$ of $0.375 \%$ levo-bupivacaine was infiltrated. For the sacral plexus block, a threshold of $0.4 \mathrm{~mA}$ was reached and $10 \mathrm{mls}$ of $0.375 \%$ levo-bupivacaine was infiltrated. She was subsequently muscle relaxed, intubated and ventilated using PCV for the procedure. Anaesthesia was maintained throughout with sevoflurane in air/oxygen mixture. Intra-operatively, the patient was haemodynamically stable requiring $2 \mathrm{~L}$ of crystalloids, $1 \mathrm{mg}$ metaraminol and $9 \mathrm{mg}$ of ephedrine. Intra-operative analgesia was minimal, with only $2 \mathrm{mg}$ of morphine administered. Post-operatively, she was managed on HDU for 3 days before discharge to the ward. Physiotherapy was commenced on day 1 post operatively. Analgesia requirement were fulfilled with regular paracetamol, no additional analgesics were requested by the patient.

\subsection{Case 3}

A 71-year-old female presented with fractured neck of left femur following a mechanical fall. Patient's medical history included severe dilated cardiomyopathy for which AICD was implanted 9 years before this episode. She also suffered with ischaemic heart disease with angina on minimal exertion. This cardiac history was on a background of three previous myocardial infarctions, atrial fibrillation, hypertension, and hyperlipidaemia. In addition to all the above, she also suffered with hypothyroidism, CKD stage 3/4 with an estimated glomerular filtration rate (eGFR) of 23-28. An echocardiogram was performed as part of her work up which demonstrated a dilated left ventricle, left ventricular dysfunction, moderate to severe mitral regurgitation and moderate tricuspid regurgitation.

Surgery was delayed because of an episode of acute left ventricular failure and possible pneumonia. Treatment with furosemide and tazocin (piperacillin-tazobactam) was commenced. Her recovery from LVF was complicated by episodes of chest pain and evidence of arterial oxygen desaturation so her treatment was continued on the coronary care unit (CCU). IV nitrate (Isoket) was added to her treatment. On day 5 post-admission, she was deemed fit for surgery by the medical team.

On the day of the surgery, the technicians from cardio-respiratory lab disabled the ICD function to allow the use of monopolar diathermy and reprogrammed the pacemaker to $\mathrm{VOO}$ at a fixed rate of $80 \mathrm{bpm}$ as per local policy. Induction of anaesthesia was performed using sevoflurane in oxygen. Spontaneous respiration was maintained throughout. Size 3 i-gel supraglottic device was inserted for airway management. After induction of anaesthesia, invasive monitoring was established with arterial cannula inserted into the radial artery and central venous access was obtained using a right internal jugular vein with a multi-lumen catheter. The patient was then turned to the right lateral position for the plexus blocks and surgery. A left lumbar plexus block $(0.4 \mathrm{~mA} 30 \mathrm{ml} 0.375 \%$ levobupivacaine) and left para-sacral block $(0.5 \mathrm{~mA} 10 \mathrm{ml}$ $0.375 \%$ levobupivacaine) were performed using a nerve stimulator. Due to complexity of the case, the procedure was performed by a senior orthopaedic surgeon and surgery lasted just under 30 minutes. An uncemented hip prosthesis was inserted uneventfully. A total of 500mls of colloid was used and $1 \mathrm{~g}$ of IV paracetamol was given for analgesia. No 
morphine or any other opioids were used. Emergence from anaesthesia was uneventful and pain scores in the recovery room were 0 (0-3) so no additional analgesia was required in the immediate post-operative period. The AICD was re-programmed, the defibrillation function was switched back on and the pacemaker was reset to VVIR at $60 \mathrm{bpm}$. The patient was transferred was discharged back to the Neck of Fracture Unit (NOFU) from the recovery ward. In the NOFU the patient was looked after by a consultant in elderly medicine and followed up by the cardiology team. The patient was mobilised on the second post op day. The analgesic requirements were minimal and met with regular IV Paracetamol initially and this was subsequently changed to oral.

\section{Discussion}

Repair of fractured neck of femur is a common operative procedure, particularly in elderly populations. Meta-analysis of randomized trials has suggested only slight advantages of regional techniques over general anaesthesia in terms of early mortality, intra-operative blood loss and thrombo-embolic complications in general populations ${ }^{[1]}$. Despite this, there remains no 'gold standard' with regards to anaesthetic management, particularly in cases where the use of central neuraxial blockade is contraindicated or avoidance of such techniques is preferable. In these three cases, we successfully carried out combined lumbar and sacral plexus blocks in addition to general anaesthesia for intra and post-op pain relief.

The sensory innervation of the hip is derived from branches of both the lumbar and sacral plexus. Lumbar plexus blockade has been shown to be reliable in blocking major branches of the lumbar plexus ${ }^{[2]}$, and the sacral plexus block has been shown to be a technically easy block to perform with a high success rate, close to $94 \%{ }^{[3]}$.

The problems associated with central neuraxial blockade in patients with severe aortic stenosis are well established ${ }^{[4]}$. In our first case, the decision was made to carry out a general anaesthetic, as per the patient's preference, combined with sacral and lumbar plexus blocks. As well as the benefits already mentioned, regional approaches have also been shown to be associated with a smaller initial decrease in mean arterial pressure compared with spinal anaesthesia using plain bupivacaine ${ }^{[5]}$.

The benefits of continuous catheter techniques have been investigated in relation to both hip arthroplasty and total knee arthroplasty. Continuous lumbar plexus block has been shown to provide improved analgesia and patient satisfaction with fewer side effects compared to systemic opioids in both hip and knee arthroplasties ${ }^{[6,7]}$. There is no evidence of clear benefit in terms of early recovery, length of stay or morphine use when using continuous versus single-injection lumbar plexus block as an adjunct to knee arthroplasty ${ }^{[10,11]}$. In our cases, general anaesthesia with lumbar and sacral plexus block was adopted as, spinal anaesthesia was contraindicated due to its vasodilatory effects and we were keen to avoid the side effects of morphine owing to the patients co-existing poor respiratory function. The option of a continuous catheter technique is not without potential side effects and therefore was not considered in these cases ${ }^{[8,9]}$. The duration of analgesia from single shot lumbar and sacral plexus lasts for at least 14-18 hours in most patients and more than 24hrs in the elderly.

In our second case, we carried out lumbar and sacral plexus blocks along side a general anaesthetic despite the patient actively taking clopidogrel. The risks associated with patients actively taking clopidogrel who may benefit from either central neuraxial blockade or peripheral nerve blockade are not well known. However, there have been significant bleeding events, including a fatal outcome, reported following lumbar plexus blocks on patients taking clopidogrel ${ }^{[12]}$. Increasing number of patients on clopidogrel therapy are presenting for urgent hip fracture surgery. Early withdrawal of clopidogrel has been shown to be associated with increased incidence of acute coronary events ${ }^{[13]}$.

Clopidogrel causes inhibition of platelet aggregation and amplification by binding to the adenylate cyclase-coupled ADP P2Y12 receptor. A recent review of epidural insertion in vascular patients taking clopidogrel attempted to address this issue but recognised the need for further prospective studies ${ }^{[14]}$. This lack of evidence has been highlighted by the 3rd 
American Society of Regional Anaesthesia (ASRA) conference on regional anaesthesia. They conservatively conclude that their consensus statements on neuraxial regional techniques should be applied to both plexus and peripheral nerve blockades, though recognising that this approach may be overly restrictive ${ }^{[15]}$. The absence of clinical guidelines relating to these issues is a reflection of the fact that more research is required in this area to guide clinical judgement.

We have used landmark techniques in all 3 cases. Although reviews of the evidence with regards to the use of ultrasound for lower limb blocks have shown a reduction in the dose of local anaesthetic used, there is not yet sufficient evidence to advise definitive recommendations regarding quality of sensory block, number of needle punctures or block duration ${ }^{[16]}$.

Dilated cardiomyopathy is defined as a large heart cavity with impaired systolic function of one or both ventricles. Anaesthetic management may be associated with a high mortality owing to cardiac complications and AICDs are an increasingly common complicating factor in patients presenting for emergency surgery. Severe dilated cardiomyopathy associated with drug resistant malignant ventricular arrhythmias is increasingly the primary reason for implantation ${ }^{[17]}$. AICDs are associated with a reduction in mortality from $66 \%$ per year to $8.5 \%$ per year in such patients. The problems encountered in patients with AICDs during surgery include shivering, fasciculation, orthopaedic instrumentation and electro-cautery interference being detected as arrhythmias and inappropriate shocks being delivered, which may induce ventricular arrhythmias depending on which period of the cardiac cycle the shock is delivered. The AICD function should therefore be deactivated prior to surgery, and arrhythmias anticipated and treated via the application of external defibrillator pads. Patients with cardiomyopathy frequently have poor cardiac reserve, and any reduction in myocardial contractility or vasodilatation can cause profound hypotension. Due to these facts, it is a rational decision to adopt a local anaesthetic technique where possible, as haemodynamic stability is vital, though there is little in the way of evidence to definitively recommend one technique over another in these patients. In this case, a lumbar and sacral plexus block in addition to general anaesthesia resulted in a good outcome with the minimal use of additional analgesia.

As can be seen from these three high-risk cases, the analgesic requirements can be met with simple oral analgesics in the immediate post-op period and most patients can be mobilised for physiotherapy by the second post-op day. The 30 day mortality in such cases remains to be high but we can ensure that the patient have good quality of recovery and they can be discharged home or into the community reducing the length of stay in the hospital.

These three cases demonstrate that lumbar and sacral plexus blocks can be a useful adjunct to general anaesthesia in selected high risk patients and provided good outcomes in terms of analgesia required, early mobilization and minimal postoperative complications.

\section{Competing interests/ conflict of interests}

The authors declare no competing interests

\section{References}

[1] Urwin SC, Parker MJ, Griffiths R. General versus regional anaesthesia for hip fracture surgery: a meta-analysis of randomized trials. British Journal of Anaesthesia. 2000; 84 (4): 450-5. http://dx.doi.org/10.1093/oxfordjournals.bja.a013468

[2] Farny J, Girard M, Drolet P. Posterior approach to the lumbar plexus combined with a sciatic nerve block using lidocaine. Can J Anaesth. 1994; 41: 486-91. http://dx.doi.org/10.1007/BF03011542

[3] Ripart J, Cuvillon P, Nouvellon E, Gaertner E, Eledjam JJ. Parasacral Approach to Block the Sciatic Nerve: A 400-Case Survey. Regional Anesthesia and Pain Medicine. 2005 March-April; 30 (2): 193-197.

[4] Stoeling RK, Dierdorf SF. Anaesthesia and co-existing disease. 3rd ed. New York: Churchill Livingstone, 1993.

[5] Visme V, Picart F, Jouan R, Legrand A, Savry C, Morin V. Combined Lumbar and Sacral Plexus Block Compared With Plain Bupivacaine Spinal Anesthesia for Hip Fractures in the Elderly. Regional Anesthesia and Pain Medicine. 2000 March-April; 25(2): 158-162. http://dx.doi.org/10.1097/00115550-200003000-00005 
[6] Siddiqui Z, Cepeda S, Denman W, Schumann R, Carr D. Continuous Lumbar Plexus Block Improved Analgesia With Fewer Side Effects Compared With Systemic Opioids After Hip Arthroplasty: A Randomized Control Trial. Regional Anaesthesia and Pain Medicine. 2007 Sept-Oct; 32(5): 393-98.

[7] Marino J, Russo J, Kenny M, Herenstein R, Livote E, Chelly J. Continuous Lumbar Plexus Block for Postoperative Pain Control After Total Hip Arthroplasty. A Randomized Control Trial.

[8] Pousman R, Mansoor Z, Sciard D. Total Spinal Anaesthetic after Continuous posterior Lumbar Plexus Block. Anesthesiology 2003; 98: 1281-2. http://dx.doi.org/10.1097/00000542-200305000-00034

[9] Ben-David B, Joshi R, Chelly J. Sciatic Nerve Palsy after Total Hip Arthroplasty in a Patient Receiving Continuous Lumbar Plexus Block. Anesth Analg. 2003; 97: 1180-2. http://dx.doi.org/10.1213/01.ANE.0000080203.58659.83

[10] Watson MW, Mitra D, McLintock TC, Grant SA. Continuous versus single-injection lumbar plexus blocks: comparison of the effects on morphine use and early recovery after total knee arthroplasty. Reg Anesth Pain Med. 2005 Nov-Dec; 30(6): $541-7$.

[11] Francis V. Salinas, MD, Spencer S. Liu, MD, Michael F. Mulroy, MD. The Effect of Single-Injection Femoral Nerve Block Versus Continuous Femoral Nerve Block After Total Knee Arthroplasty on Hospital Length of Stay and Long-Term Functional Recovery Within an Established Clinical Pathway. Anesth Analg. 2006; 102: 1234-9. http://dx.doi.org/10.1213/01.ane.0000198675.20279.81

[12] Maier C, Gleim M, Weiss T, Stachetzki U, Nicolas V, Zenz M. Severe bleeding following lumbar sympa- thetic blockade in two patients under medication with irreversible platelet aggregation inhibitors. Anesthesiology. 2002; 97: 740-743. http://dx.doi.org/10.1097/00000542-200209000-00032

[13] Collyer T.C, Reynolds H.C, Truyens E, Kilshaw L. Perioperative management of clopiogrel therapy:the effect on in-hospital cardiac morbidity in older patients with hip fractures. Br. J. Anaesth. 2011; 107 (6): 911-915. http://dx.doi.org/10.1093/bja/aer288

[14] Osta WA, Akbary H, Fuleihan SF. Epidural analgesia in vascular surgery patients actively taking clopidogrel. British Journal of Anaesthesia. 2010; 104 (4): 429-32 http://dx.doi.org/10.1093/bja/aeq029

[15] Horlocker T, Wedel D, Rowlingson J, Enneking F, Kopp S, Benzon H, et al. Regional Anesthesia in the Patient Receiving Antithrombotic or Thrombolytic Therapy: American Society of Regional Anesthesia and Pain Medicine Evidence-Based Guidelines (Third Edition). Regional Anesthesia and Pain Medicine. 2010 Jan-Feb; 35(1): 64-101. http://dx.doi.org/10.1097/AAP.0b013e3181c15c70

[16] Salinas FV. Ultrasound and review of evidence for lower extremity peripheral nerve blocks. Reg Anesth Pain Med. 2010; 35(suppl 1): S16YS25.

[17] Bardy GH, Lee KL, Mark DB, et al. Amiodarone or an implantable cardioverter-defibrillator for congestive heart failure. N Engl J Med. 2005; 352: 225-237. http://dx.doi.org/10.1056/NEJMoa043399 